\title{
Gravitation, rotating bodies, and their relative velocities
}

Boris Lagutin

Funding: The author(s) received no specific funding for this work.

Potential competing interests: The author(s) declared that no potential competing interests exist.

\section{Abstract}

This preprint contains the author's analysis of rotational motions of bodies and gravitation based on the classical mechanics formulas. The analysis discovers some contradictory points in classical and orbital mechanics. It lead the author to conclude that gravitational interactions between bodies may depend on their relative velocities. Moreover, gravitational interactions may contain an unknown effect which is relative. The preprint describes an experiment which may prove that gravitational interactions depend on velocities (directions and magnitudes) of rotating bodies relatively to each other.

Classical mechanics contains the description of forces acting at the very top of the Ferris wheel movement. The formula of the net force $\boldsymbol{F}_{\text {net }}$ for the Ferris wheel motion at the very top is the following if a direction of a normal force $\boldsymbol{N}$ (upwards) is chosen as positive [1]:

$$
F_{\text {net }}=-m a_{C}=N-m g,(1)
$$

where $\boldsymbol{a}_{\boldsymbol{c}}$ is a centripetal acceleration, $\boldsymbol{m}$ is a mass of a body of a human, $\boldsymbol{g}$ is a gravitational acceleration, see Figure 1 .

\section{Ferris wheel at point $A$ (on the very top)}

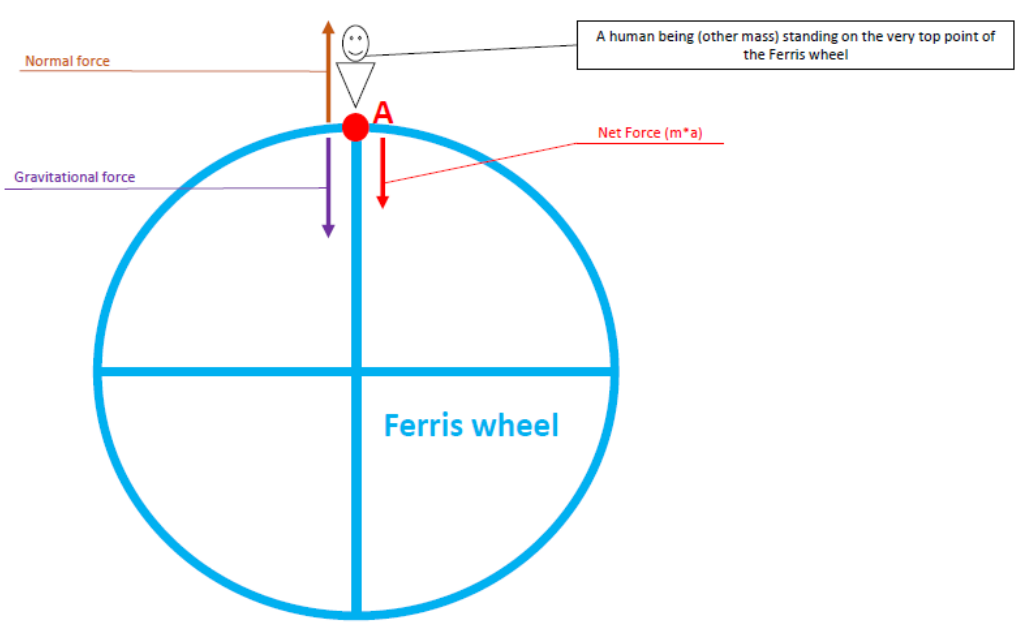

If an angular velocity increases, it means that a centripetal acceleration $\boldsymbol{a}_{\boldsymbol{c}}$ increases based on the following formula [2]:

$$
a_{c}=\frac{v^{2}}{r} \text {, (2) }
$$

where $\boldsymbol{v}$ is a tangential speed, $\boldsymbol{r}$ is a radius of rotation of a body. Hence, a magnitude of the net force $\boldsymbol{m a}_{\boldsymbol{c}}$ increases if a centripetal acceleration increases. Based on the formula (1), one can build a formula for a magnitude of a normal force $\boldsymbol{N}$

$$
N=\left|-m a_{c}+m g\right| \text {, (3) }
$$

that defines a normal force depending on a centripetal acceleration. As a result, the larger magnitude of a centripetal acceleration $\left|\mathbf{a}_{\boldsymbol{c}}\right|$ becomes over a magnitude of gravitational acceleration $|\boldsymbol{g}|$ the larger normal force becomes in according to the formula (3). In practice, humans feel that they become a bit lighter on the top of the Ferris wheel motion [1]. Moreover, if a magnitude of $\boldsymbol{m a}_{\boldsymbol{c}}$ becomes equal to a magnitude of $\boldsymbol{m g}$, then humans can experience some kind of weightlessness on the top of the Ferris 
wheel motion. But, classical mechanics does not tell what happens if a centripetal acceleration becomes larger and larger than a gravitational force. Should a human, who rides a Ferris wheel, fly up in this case? Definitely, It would not happen. Then, what physical property can a normal force $\boldsymbol{N}$, which is larger than a gravitational force $\boldsymbol{m g}$, be related to? The author will try to answer later in this article. For now, let's consider another real situation.

While sitting in a laundromat, the author watched and analyzed motions of cotton clothing which was rotating in a washing machine steel barrel. The barrel is rotated very fast to pull water out of the clothing pressing the latter to the barrel internal surface. The author tried to understand what causes a pressure between the clothing and the barrel internal surface at the very top of the uniform circular motion at a high angular speed (magnitude of angular velocity). The author analyzed all possible forces, accelerations, and velocities based on classical mechanics, see Figure 2.

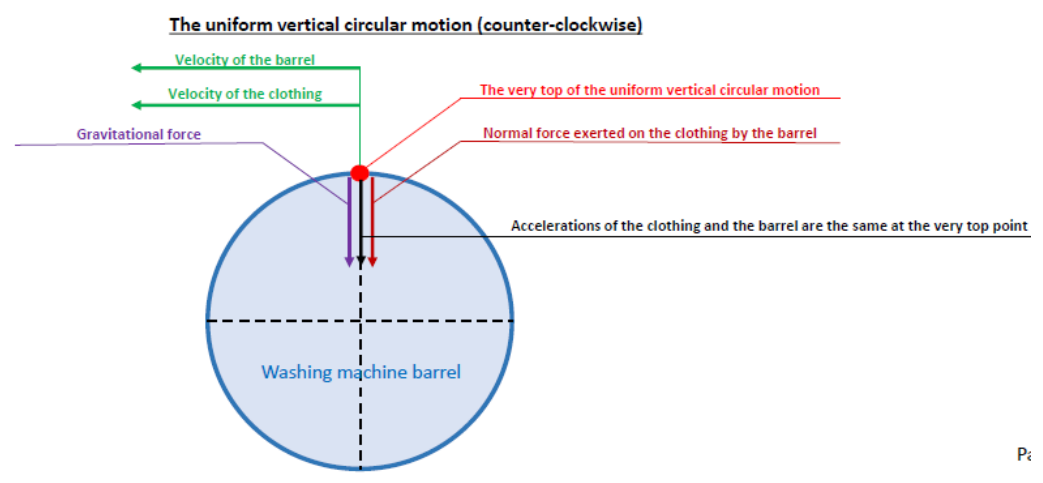

The higher angular speed becomes the larger pressure becomes. The clothing and the barrel have the same angular velocity and the same centripetal acceleration during an infinitesimal moment at the very top. In according to Newton's third law, there must be a force which acts against a normal force $\boldsymbol{N}$ exerted on the clothing by the barrel internal surface if there is the pressure as it is in the Ferris wheel rotation [1]. However, the classical mechanics formula of the net force $\boldsymbol{F}_{\text {net }}$ in the vertical plane does not have any force acting against the normal force at the very top of the washing machine barrel motion:

$$
-F_{\text {net }}=-m a_{c}=-N-m g,(4)
$$

where $\boldsymbol{m}$ is a mass of the clothing, $\boldsymbol{a}_{\boldsymbol{c}}$ is a centripetal acceleration, $\boldsymbol{g}$ is a gravitational acceleration. Notice that all the terms in the formula (4) are negative because the vectors of all these forces point at the same direction (downwards), see Figure 2. To create a pressure between two bodies (masses), it is necessary to have at least two forces acting against each other. For example, a book, which is located on a table, exerts a gravitational force $\boldsymbol{m g}$ on the table, and the table exerts a normal force $\boldsymbol{N}$ on the book, see Figure 3.

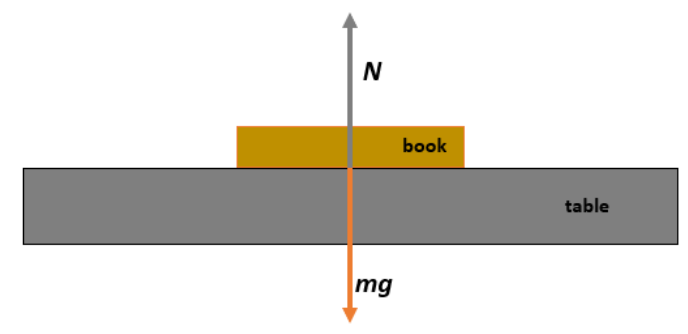

The formula of the net force for the system "book-table" contains a gravitational force $\boldsymbol{m g}$ acting against a normal force $\boldsymbol{N}$ [3]:

$$
F_{\text {net }}=N-m g,(5)
$$

where $\boldsymbol{m}$ is a mass of the book. Evidently, there is not a force acting against a normal force in the formula (4). Therefore, it is unclear how the pressure between the clothing and the barrel internal surface appears at a high angular speed. The only physical property of the clothing which can participate in creating the pressure is its inertia. Though, the moment of Inertia of the clothing $\boldsymbol{I}$ is defined by the following formula for a point mass [4]:

$$
I=m r^{2},(6)
$$

where $\boldsymbol{m}$ is a mass of the clothing, $\boldsymbol{r}$ is a radius of rotation of the clothing (from a rotational center to a center of the clothing mass). However, the moment of Inertia of the clothing is always the same independently of the clothing angular speed. Hence, the moment cannot participate in creating the growing pressure. Next, consider the motion of the system "clothing-barrel" in a bit more detail.

The green arrows symbolize the vectors of the tangential speed (counterclockwise rotation) of the clothing and of the barrel starting from the very top (point A), see Figure 4. 


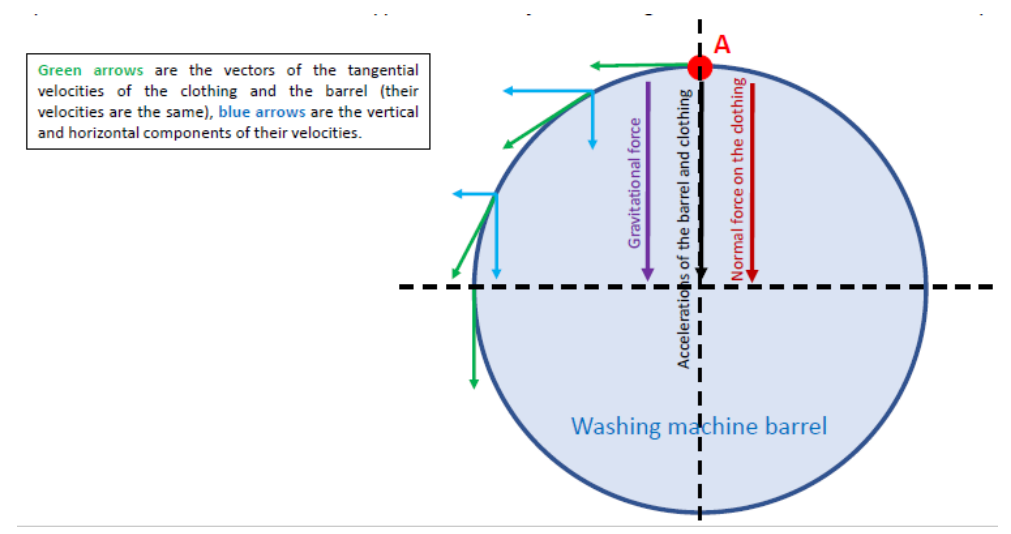

The vertical components of the velocity of the clothing and of the barrel are zeros at a point $A$. The vertical component of the clothing velocity grows downwards starting from a point $A$. The horizontal components of the clothing velocity decrease starting from a point $A$. At a point A, a gravitational force, the clothing acceleration, and a normal force exerted by the barrel on the clothing point downwards. The classical mechanics formulas, which describe the system "clothing-barrel", do not contain any force acting opposite to the normal force. Based on Newton's third law, a force opposite to the normal force is needed to create a pressure between the clothing and the barrel internal surface at a point A. It allows to state that there is an unknown force or an unknown physical property which participates in creating the pressure at a point A. However, such a force (property) does not push the clothing up, it just prevents the clothing from bouncing the barrel internal surface. Then, what pushes the clothing to the surface stronger and stronger to create a higher pressure at higher speeds? Let's analyze this point in detail.

For example, if you rotated a ball suspended by a spring faster and faster, the spring would be stretched further and further. So if the ball was released from the spring, the ball would fly straightly and the spring would return to its initial state (length). The spring is stretched more and more at higher speeds because the ball strives to fly straightly faster and faster at every point of its spiral trajectory. And, an unknown force (effect) is assumed to just prevent the ball and the spring from returning back. The fly and an unknown force (effect) happen simultaneously.

A next point for an analysis is orbital mechanics. Based on current physics, small bodies orbit the Earth because of free fall. That is, they eternally freely fall around the Earth after they enter their orbital trajectories. For example, a rocket enters its orbital trajectory at a particular tangential velocity and begins to eternally freely fall [5] because its trajectory correlates with the Earth shape. Simply saying, a rocket or another orbiting body does not have time to reach the Earth surface, so it eternally freely falls. This explanation is commonly given at schools, colleges, and universities. It seems to be consistent for students and teachers. However, let's take a deep look at it. The only force, which pulls orbiting bodies down to the Earth surface, is the Earth gravitational force. The gravitational force pulls the bodies approximately towards the Earth center because the Earth is approximately spherical. Assume that the Earth is a perfect sphere, then the gravitational force pulls the bodies exactly to the center, see Figure 5 .

\section{The empirical fact is that the gravitational force is always pulling mass(es) towards the Earth's center}

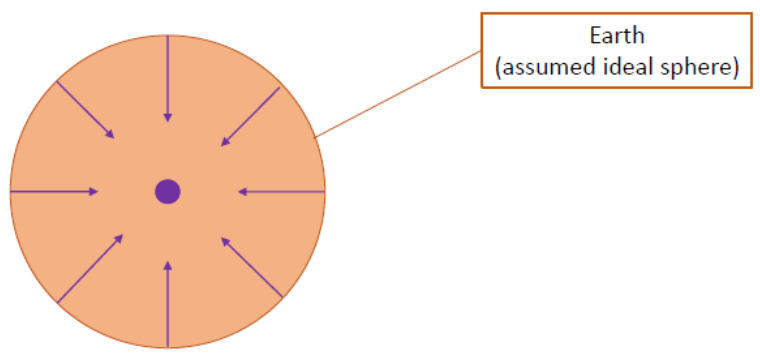

Next, consider the following three situations of projectile motion of a body. Assume that all these projectile motions happen without air resistance. Distances and heights in the situations are calculated in according to the projectile motion simulation website [6]. So, the first situation is a projectile motion of a body at a distance of about 10 meters and at a height of about 5 meters, see Figure 6. 
1. First situation

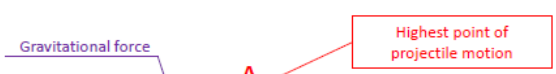

To cover the distance about 10 meters, a body has to be fired (thrown) at an approfirate initial speed at a particular angle to the Earth surface where the gravitational acceleration is about 9.81 meters per second squared. After a body reacbes 0 man mun helght about 5 meters, it starts to freely fall to the Earth surface accelerating at the rate 9.81 meters per second squared.

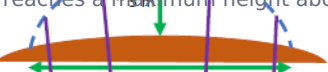

In the second situation, a body must be fired (thrown) at an appropriate initi spoed a particular angle to the Earth surface in order to cover the distance about 1000 meters, see Figure 7.

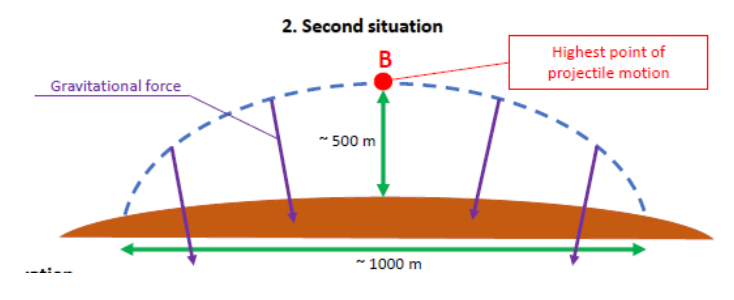

After a body reaches a maximum height about 500 meters, it starts to freely fall to the Earth surface accelerating at the rate 9.81 meters per second squared.

In the third situation, a body must be fired (thrown) at an appropriate initial speed at a particular angle to the Earth surface in order to cover the distance about 836,000 meters (836 kilometers), see Figure 8.

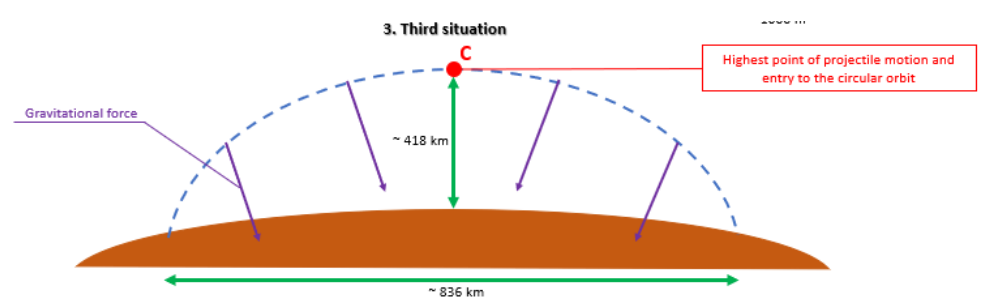

After a body reaches a maximum height about 418,000 meters (418 kilometers), it starts to freely fall to the Earth surface accelerating at the rate of slightly less than 9.81 meters per second squared. It is an unrealistic situation nowadays because one barely finds a cannon that is able to fire some body to this height.

When a body begins to freely fall after reaching its projectile trajectory maximum height, a distance between a body and the Earth surface (or the Earth center) begins to decrease at all the three situations. It means that the distance decreases radially, in other words, it decreases along the Earth radius. Notice that the projectile trajectory in Figure 8 results from firing a body; in other words, a body is given an only initial impulse. Therefore, a spacecraft (rocket) trajectory before a point $\mathbf{C}$ is different from the trajectory drawn in Figure 8 because a spacecraft (rocket) is given a continuous thrust by its engines before a point $\mathbf{C}$. Therefore, a spacecraft trajectory looks like a trajectory drawn in Figure 9, where $\boldsymbol{h}$ is the height 418 kilometers above the Earth surface, $\mathbf{C}$ is a point of a spacecraft entry in its orbital trajectory, $\mathbf{S}$ is supposed to be a point of a contact of a spacecraft and the Earth surface.

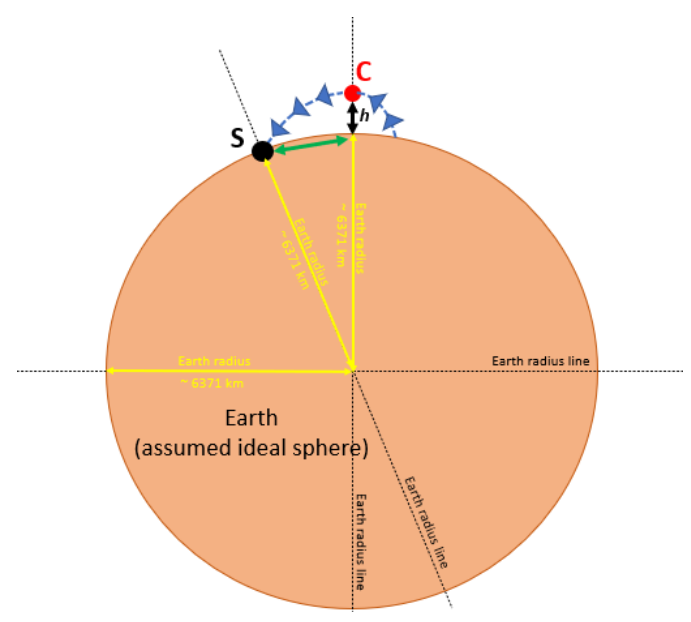

Notice that a spacecraft trajectory after a point $\mathbf{C}$ should be a regular projectile trajectory as it freely falls (no any thrust is given after a point $\mathbf{C}$ ). However, it does really not happen if a spacecraft (rocket) enters its orbital trajectory at an appropriate speed. In other words, a spacecraft (rocket) begins to freely fall eternally if it enters its projectile trajectory at an appropriate speed [7]. If no any external forces decrease a spacecraft's speed, it can really orbit the Earth eternally [8]. 
Now consider the situation pictured in Figure 9 for a spacecraft entering its orbital trajectory at a point C based on the real data (taken from Wikipedia) of the International Space Station. It is necessary to confirm whether or not a spacecraft (or even the International Space Station) can eternally freely fall or it should fall down and touch the Earth surface at a point $\mathbf{S}$ based on the real data calculations, even though it keeps an appropriate speed to stay at its orbit.

The International Space Station (ISS) as well as a spacecraft or any other body can keep its orbital trajectory only if ISS (spacecraft, body) flies at a particular speed [7] ; otherwise, it begins to change its orbit [8,9]. If the speed of ISS (spacecraft) goes down to a particularly low speed, then ISS (spacecraft) cannot orbit the Earth. That is, it simply falls on the Earth surface as it happens in the projectile motion from a point C [10], see Figure 8. Wikipedia contains the following data of ISS: 1) the Earth radius is about 6,371 kilometers (6,371,000 meters); 2) the Earth gravitational acceleration near the Earth surface is about 9.81 meters per second squared; 3 ) the Earth gravitational acceleration at the height of $418 \mathrm{~km}$ is 8.829 meters per second squared which is $90 \%$ of that near the Earth surface; 4 ) the ISS orbital height at perigee is about 418 kilometers; 5) the ISS orbital speed is about 7.66 kilometers per second or 7,660 meters per second; 6 ) the ISS orbital period (time to make a full ellipse around the Earth) is about 92.65 minutes or 5,559 seconds [11, 12]. The author decided to use a mean gravitational acceleration which is

$$
g_{\text {mean }}=\frac{9.81^{\frac{m}{s^{2}}}+8.829^{\frac{m}{s^{2}}}}{2}=9.3195^{\frac{m}{s^{2}}} . \text { (7) }
$$

Based on the Wikipedia data, make some calculations for a spacecraft (rocket) counter-clockwise entering its orbital trajectory of the height 418 kilometers, see Figure 8. Notice that the ability to stay at an orbital trajectory does not depend on a mass of a spacecraft (rocket), but the latter velocity plays a key role.

All orbiting bodies are radially pulled down to the Earth at the gravitational acceleration. A spacecraft (rocket), which enters its orbital trajectory at the height 418 kilometers, begins to freely fall from a point $\mathbf{C}$ based on classical or orbital mechanics, see Figure 9 . Hence, the classical mechanics formula for distance d, which a spacecraft (rocket) should freely fall, can be used to count how low a spacecraft (rocket) should fall radially beginning from a point $\mathbf{C}$ [13]:

$$
d \approx \frac{g_{\text {mean }} t^{2}}{2}
$$

A tangential speed of and a vertical (falling) speed of a spacecraft are calculated independently from each other. An orbital height of a spacecraft (distance from a spacecraft to the Earth surface) is about $418 \mathrm{~km}(418,000 \mathrm{~m})$. Therefore, time $\boldsymbol{t}$, which it takes a spacecraft to touch the Earth surface, can be derived from the formula $(8)$ :

$$
t=\sqrt{\frac{2 d}{g_{\text {mean }}}}
$$

where $\boldsymbol{t}$ is time in seconds, hence:

$$
t=\sqrt{\frac{418,000 m 2}{9.3195^{\frac{m}{s^{2}}}}} \approx 299.51 s
$$

Now, it is possible to calculate a length $\boldsymbol{L}$ of a spacecraft trajectory from a point $\mathbf{C}$ to a point $\mathbf{S}$, see Figure 9 , by multiplying a spacecraft tangential speed and time $\boldsymbol{t}$ :

$$
L=7,660^{\frac{m}{s}} 299.51 s=2,294,246.60 m \approx 2,294.00 \mathrm{~km} \text {. (11) }
$$

The orbital period $\mathbf{T}$ of the International Space Station as well as a spacecraft, which orbits the Earth at the height $418 \mathrm{~km}$, is about 92.65 minutes ( 5559 seconds). It means that a spacecraft or the International Space Station flies the following total orbital length $\boldsymbol{L}_{\text {total }}$ making a full ellipse (almost a circle):

$$
L_{\text {total }}=5559.00 \mathrm{~s} 7,660.00^{\frac{m}{s}}=42,581,940.00 m \approx 42,582.00 \mathrm{~km} . \text { (12) }
$$

So if divide the total orbital length by four, one gets a quarter of the orbital trajectory:

$$
\frac{L_{\text {total }}}{4}=10,645,485.00 \mathrm{~m} \approx 10,646.00 \mathrm{~km} . \text { (13) }
$$

It is easy to figure out that $\boldsymbol{L}$ is about 4.64 times less than a quarter of $\boldsymbol{L}_{\text {total }}$. It should be underscored that $\boldsymbol{L}$ is about 4.64 times less just a quarter of the total orbital trajectory (not the total orbital trajectory). To sum up, a spacecraft or the International Space Station should freely fall down and contact the Earth surface at a point $\mathbf{S}$, see Figure 9, in just 299 seconds since their entry in their orbital trajectory at a point $\mathbf{C}$ at the height 418 kilometers. Based on the calculations and the fact that the distance (height) between a spacecraft (International Space Station) and the Earth surface can only decrease radially, a spacecraft (International Space Station) should fall down on the Earth surface. However, it does not happen in reality. The latter means that a body, which enters its orbital trajectory at a particular velocity, which is specific for every orbital trajectory, experiences an unknown force (effect) that prevents such a body from falling down to the Earth surface. As it is seen from the analysis, such an unknown force (effect) is related to a body relative velocity. Relative velocity means a body velocity relative to another body. The latter is the Earth in this paper.

If assume that a mass $\boldsymbol{m}_{\text {spacecraft }}$ is 10,000 kilograms, then an unknown force (effect), which prevents a spacecraft (rocket) from falling down, should be equal to or more than a gravitational force $\boldsymbol{F}_{\text {gravity }}$ exerted by the Earth on a spacecraft (rocket). $\boldsymbol{F}_{\text {gravity }}$ at a spacecraft orbit (418 $\mathrm{km}$ ) is about $90 \%$ of a gravitational force near the Earth surface:

$$
F_{\text {gravity }}=10,000.00 \mathrm{~kg} 9.81^{\frac{m}{s^{2}}} 0.90=88,290.00 \mathrm{~kg}^{\frac{m}{s^{2}}} . \text { (14) }
$$

A spacecraft or the International Space Station rotates around the Earth at the same orbital trajectory. It means that they have the same centripetal acceleration. Remind that centripetal acceleration only depends on a tangential speed and a rotational radius. Analyzing the equation (3), it can be assumed that an unknown force (effect) should be equal to $\boldsymbol{m} \boldsymbol{a}_{\boldsymbol{c}}$, where $\boldsymbol{a}_{\boldsymbol{c}}$ is centripetal acceleration of a spacecraft, $\boldsymbol{m}$ is a spacecraft mass, when a spacecraft is at its circular or elliptical orbital trajectory. "When a 
spacecraft is at its circular or elliptical orbital trajectory" means that a distance between a spacecraft and the Earth surface (the Earth center) does not decrease as it happens in the projectile motion after a point C, see Figure 8. A spacecraft tangential speed $\boldsymbol{V}$ is the same as that of the International Space Station (ISS) $\sim 7,660$ meters per second. A rotational radius $\boldsymbol{R}_{\boldsymbol{s}}$ of a spacecraft and ISS is:

$$
R_{S} \approx 6,371,000 m+418,000 m \approx 6,789,000 m \text {. (15) }
$$

Hence, $\boldsymbol{m a}_{\boldsymbol{c}}$ of a spacecraft can be calculated based on the formula (2):

$$
m a_{c} \approx m^{\frac{V^{2}}{R_{s}}} \approx 10,000.00 \mathrm{~kg}^{\frac{\left(7,660.00^{\frac{m}{s}}\right)^{2}}{6,789,000.00 m}} \approx 86,427.00 \mathrm{~kg}^{\frac{m}{\mathrm{~s}^{2}}}
$$

Taking into account that $90 \%$ of the gravitational force at the orbit is just an approximation, compare $\boldsymbol{F}_{\text {gravity }}$ and $\boldsymbol{m a}_{\boldsymbol{c}}$ of a spacecraft, it is clear that they correlate with each other:

$$
88,290.00 \mathrm{~kg}^{\frac{m}{s^{2}}} \approx 86,427.00 \mathrm{~kg}^{\frac{m}{\mathrm{~s}^{2}}} .(17)
$$

If the author's assumption of an unknown force (effect) is right, then it should be right for all rotating bodies. This statement is confirmed by the formulas and the observations of the Ferris wheel motion when humans may experience some kind of weightlessness on the top of a Ferris wheel or feel lighter. Though, it should be mentioned that an unknown force (effect) is relative. One example of relative nature of an unknown force (effect) is: astronauts and other bodies in the International Space Station. The astronauts can easily move any bodies towards the Earth center as well as any other direction, so they all have the same velocities. That is, an unknown force (effect) does not act against them. It is important to notice that the system "clothing - barrel" is totally different from the system "astronauts - other bodies" in ISS.

Following the analysis of the rotational motions and the orbital mechanics, the author designed an experiment to discover a gravitational interaction dependence on a relative velocity of a body. The main components of an experimental device are rotational masses (stainless steel sticks) each of which contains a stainless steel ball at one end. Another end of each stick is firmly assembled to a programmable stepper electromotor, see Figure 10.

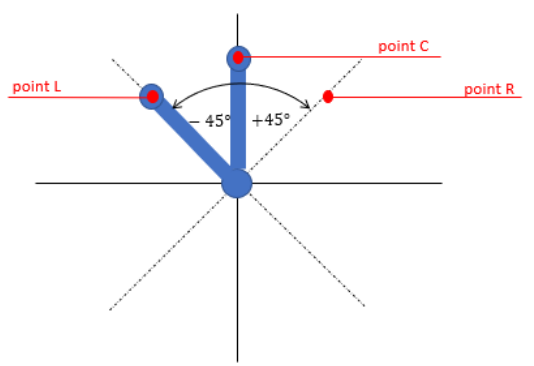

The sticks rotate at different phases of 90 degrees each at opposite directions. The sticks are firmly connected to each other by bearings, Figure 11.

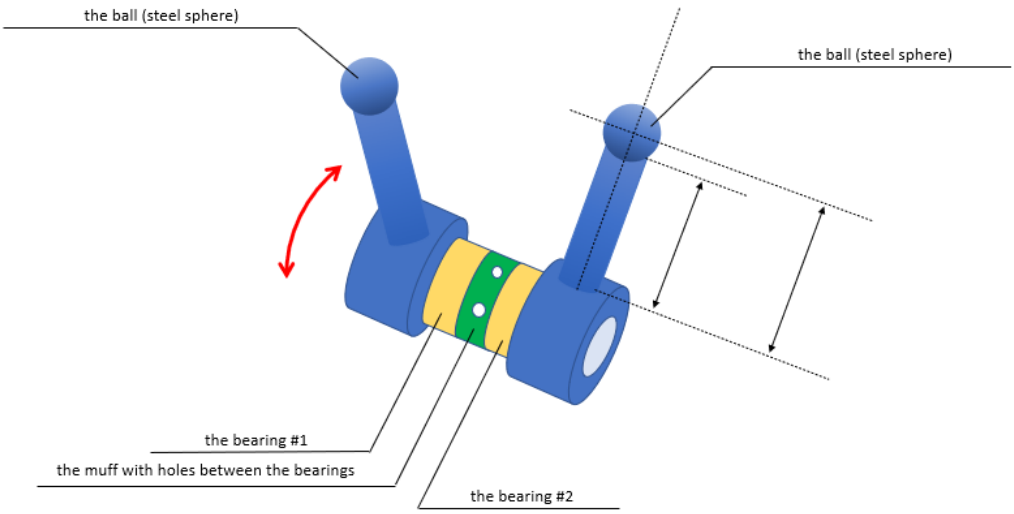

The sticks with the two electromotors are assembled on a vertically movable rigid platform. The platform may move up and down freely on wheels. The electromotors are manipulated by a computer via a controller, see Figure 12 


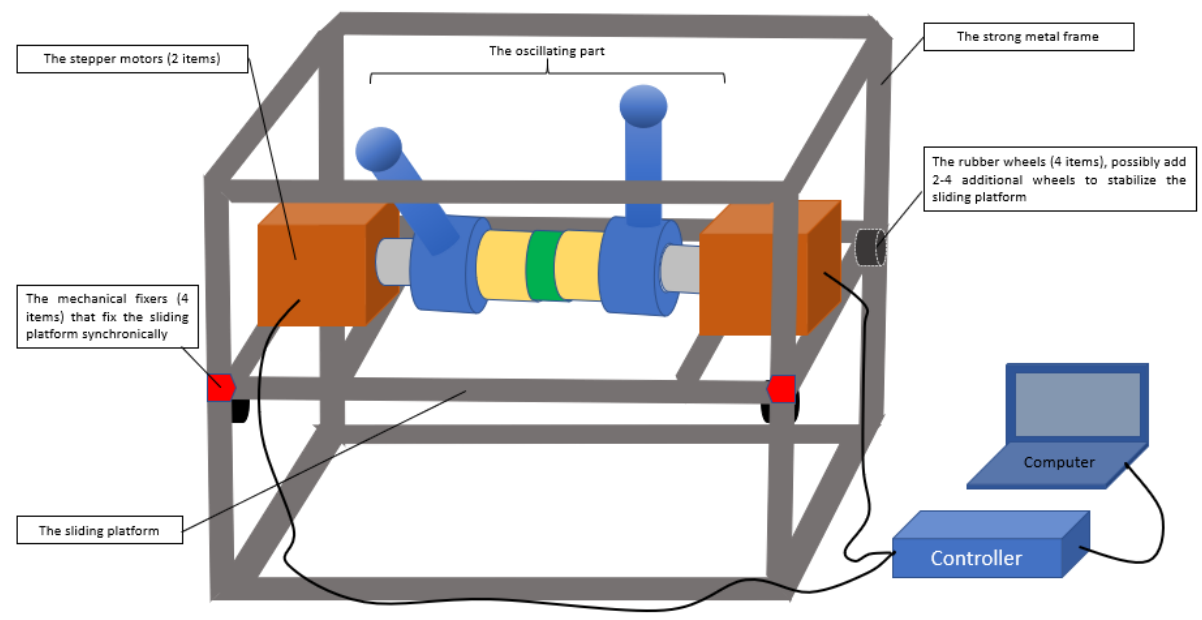

When one stick starts to rotate counter-clockwise from a point $\mathbf{C}$ to a point $\mathbf{L}$, another stick starts to rotate clockwise from a point $\mathbf{L}$ (left side) to a point $\mathbf{R}$ (right side). When the first stick reaches a point $\mathbf{L}$, it stops and begins to rotate clockwise to a point $\mathbf{R}$. When the second stick reaches a point $\mathbf{R}$, it begins to rotate counter-clockwise to a point L. In this way, they make a full cycle. The cycle repeats as many time as necessary.

A mass of each ball $\boldsymbol{m}_{\boldsymbol{b a l l}}$ must be enough to create a centripetal force $\boldsymbol{m}_{\boldsymbol{b a l l}} \boldsymbol{a}_{\boldsymbol{c}}$ which is equal to or more than a weight of the platform with the electromotors, the sticks, and other components. It is important because a maximum rotational speed of electromotors is limited. Hence, it is impossible to infinitely increase the speed in order to get a larger centripetal force. On the other side, it may be problematic for stepper electromotors to rotate and to stop the very heavy sticks at necessary experimental speeds. The experimental device seems to be simple; however, there are some practical limitations: time to stop the sticks at points $\mathbf{L}$ and $\mathbf{R}$, a maximum speed, a maximum torque of stepper electromotors etc. For example, an average weight of a small standard stepper electromotor is about 8 Newtons. It means that a weight of the platform with the sticks, the two electromotors, and other components may be about 30 Newtons. Consequently, $\boldsymbol{m}_{\boldsymbol{b a l l}} \boldsymbol{a}_{\boldsymbol{c}}$ may be equal to or more than 30 Newtons. So if a maximum speed of a stepper electromotor is 2,000 rotations per minute (RPM), then a tangential speed of the ball $\boldsymbol{V}_{\boldsymbol{b a l l}}$ is:

$$
v_{\text {ball }}=\frac{2,000 R P M \pi 2 r_{\text {ball }}}{60 s}=\frac{4,000 \pi}{60} r_{\text {ball }} \frac{\mathrm{m}}{\mathrm{s}},
$$

where $\boldsymbol{r}_{\boldsymbol{b a l l}}$ is a distance from the ball to a center of its rotation in meters, hence $\boldsymbol{a}_{\boldsymbol{c}}$ based on the equation (2):

$$
a_{c}=\frac{v_{\text {ball }}^{2}}{r_{\text {ball }}}=\frac{\left(4,000 \pi r_{\text {ball }}\right)^{2}}{(60 s)^{2} r_{\text {ball }}}=\frac{4,000^{2}}{60^{2}} \pi^{2} r_{\text {ball }} \frac{m}{s^{2}} .
$$

Therefore, a formula of a centripetal force $\boldsymbol{m}_{\boldsymbol{b a l l}} \boldsymbol{a}_{\boldsymbol{c}}$ becomes:

$$
m_{\text {ball }} a_{c}=m_{\text {ball }} \frac{4,000^{2}}{60^{2}} \pi^{2} r_{\text {ball }} k g^{\frac{m}{s^{2}}} . \text { (20) }
$$

The necessary parameters of the sticks (mass and length) with the balls can be calculated in according to the equations (18), (19), and (20). Also, it should be counted that the balls come between points $\mathbf{L}$ and $\mathbf{C}$, and $\mathbf{C}$ and $\mathbf{R}$. In other words, they are not perpendicular to the Earth surface between these points. It means that an unknown force (effect) is assumed to be weaker than it is exactly at a point $\mathbf{C}$

Conclusion

If the platform stops to slide down when the sticks are swinging at an appropriate speed, it means that an assumed unknown force (effect) is confirmed. The force (effect) is also confirmed if the platform slides down slower than it does when the sticks are motionless. However, it is necessary to pay attention to how vibrations influence the platform movement (friction and normal forces between the wheels and the frame).

In case of the experimental confirmation of an unknown force (effect), it appears that gravitational interactions between bodies may depend on their relative velocities. The gravitation dependence on velocities was also assumed by Professor Sergey V. Siparov [14].

Discussion

The experiment described in this paper resulted from a long time discussion with Professor Joe Wolfe who defended classical mechanics.

Acknowledgement

The author thanks Professor Joe Wolfe for his remarks and time.

References:

1. https://www.wired.com/story/why-do-you-feel-lighter-at-the-top-of-a-ferris-wheel/ 
2. https://www.khanacademy.org/science/physics/centripetal-force-and-gravitation/centripetal-acceleration-tutoria/a/what-is-centripetal-acceleration

3. http://hadron.physics.fsu.edu/ crede/TEACHING/PHY2048C/Calendar/W5_D2/lecture-tension.pdf

4. https://www.feynmanlectures.caltech.edu/l_19.html

5. https://www.uh.edu/engines/epi1534.htm

6. https://phet.colorado.edu/sims/html/projectile-motion/latest/projectile-motion_en.html

7. https://www.grc.nasa.gov/www/k-12/rocket/rktrflght.html

8. https://www.faa.gov/about/office_org/headquarters_offices/avs/offices/aam/cami/library/online_libraries/aerospace_medicine/tutorial/media/lll.4.1.5_Maneuvering_in_Space.pc

9. https://www.wired.com/2010/11/changing-orbits-and-changing-speed/

10. https://en.wikipedia.org/wiki/Orbital spaceflight

11. https://en.wikipedia.org/wiki/Earth

12. https://en.wikipedia.org/wiki/International_Space_Station

13. https://www.khanacademy.org/science/physics/one-dimensional-motion/kinematic-formulas/v/deriving-displacement-as-a-function-of-time-acceleration-and-initial-velocity

14. Siparov V. Sergey (2009) Anisotropic geometrodynamics in cosmological problems, www.arXiv.org, Cornell University, August 2009: https://arxiv.org/abs/0809.1817v3 